\title{
Second-Order Dielectric Constant of a Copolymer of Vinylidene Fluoride and Trifluoroethylene (52 : 48 Mole Ratio)
}

\author{
Susumu IKeDA, Hideshige SuzUKI, Kiyohito KoYAMA, \\ and Yasaku WADA \\ Department of Polymer Materials Engineering, Faculty of Engineering, \\ Yamagata University, Jonan 4-3-16, Yonezawa 992, Japan
}

(Received December 8, 1986)

\begin{abstract}
The second-order dielectric constant $\varepsilon_{2}$ of a copolymer of vinylidene fluoride and trifluoroethylene ( $52: 48$ in mole) was measured by means of the difference frequency method. $\varepsilon_{2}$ is related to spontaneous polarization $P_{\mathrm{s}}$ and the linear dielectric constant $\varepsilon_{1}$ through a thermodynamic representation of free energy. The relation explains the observed temperature dependence of $\varepsilon_{2}$. $\varepsilon_{2}$ was found to be proportional to the remanent polarization $P_{\mathrm{r}}$. This is interpreted by the model that the ferroelectric crystal is composed of parallel and antiparallel domains. $\varepsilon_{2}$ in the crystal was deduced from that of the whole polymer on the assumption of the ellipsoidal crystal model.

KEY WORDS Nonlinear Dielectric Constant / Second-Order Dielectric Constant / Ferroelectric Polymer / Vinylidene Fluoride-Trifluoroethylene Copolymer /
\end{abstract}

Poly(vinylidene fluoride) (PVDF) and copolymers of vinylidene fluoride (VDF) and trifluoroethylene (TrFE) possess piezoelectric, pyroelectric and ferroelectric properties. The ferroelectricity and the ferroelectric-toparaelectric phase transition of the copolymers have been studied by D-E (electric displacement-electric field) hysteresis, ${ }^{1,2}$ polarization reversal switching current ${ }^{3,4}$ and anomalies of various quantities at the transition. ${ }^{5}$ Molecular conformations and crystal structures of the copolymers in the vicinity of the transition have been investigated by X-ray diffraction and IR absorption. ${ }^{6,7}$ Since the ferroelectric polarization is deduced thermodynamically from the free energy as a function of polarization $P$ including higher order terms as $P^{4}$ and $P^{6}$, higher order dielectric constants with an appreciable magnitude are expected to appear in the ferroelectric polymers. These constants should be closely related to the coefficients of $P^{4}$ and $P^{6}$ terms in the free energy.

In the first part of this paper, an experimental system to obtain the second-order dielectric constant is described. The system is essentially similar to that reported by Hayakawa and his coworkers. $^{8}$

Secondly we measured the temperature dependence of second-order dielectric constant of a VDF-TrFE copolymer with VDF fraction of $52 \mathrm{~mol} \%$ which exhibits a ferroelectric phase transition at $c a .70^{\circ} \mathrm{C}$. Since the second order dielectric constant of the copolymer vanishes in the paraelectric state with a symmetry center, we could determine the transition temperature from its temperature dependence.

Thirdly, the dependence of the second order dielectric constant on the remanent polarization $P_{\mathrm{r}}$ of samples was observed to be discussed on the assumption that the system has a multi-domain structure. Since the ferroelectric polymer includes paraelectric noncrystalline regions, we must employ a com- 
posite model for deducing the intrinsic properties of a crystal from the results observed for the whole polymer. The relation between the observed ferroelectricity and the intrinsic property of the crystal is discussed on the basis of a simple ellipsoid model. The second order dielectric constant of crystal is evaluated by this model.

\section{EXPERIMENTAL}

\section{Materials}

Samples were cut out from a sheet of VDFTrFE copolymer ( $52 \%$ VDF content) manufactured by Daikin Kogyo Co. The thickness of samples ranged around $45 \mu \mathrm{m}$. Prior to measurements, the samples were annealed at $130^{\circ} \mathrm{C}$ for $30 \mathrm{~min}$ and then poled under an electric field of $90 \mathrm{MVm}^{-1}$ at $20^{\circ} \mathrm{C}$ for $30 \mathrm{~min}$. The linear dielectric constant and thermally stimulated current were measured with a Rheolograph-Solid (Toyo Seiki Co). The remanent polarization at each temperature was calculated by integrating the thermally stimulated current with respect to time.

\section{Measurement of Second-Order Dielectric Constant}

The electric displacement $D$ of a ferroelectric material is expanded in a power series of electric field $E$ as

$$
D=P_{\mathrm{s}}+\varepsilon_{1} E+\varepsilon_{2} E^{2}+\varepsilon_{3} E^{3}+\cdots
$$

where $\varepsilon_{n}$ is the $n$-th order dielectric constant.

When an electric field given by eq 2 is applied to a sample, nonlinear responses at $2 f_{1}, 2 f_{2}, f_{1}-f_{2}, f_{1}+f_{2}, \cdots$ etc. arise from the higher order terms in eq 1 in addition to linear responses at $f_{1}$ and $f_{2}$.

$$
E=E_{0}\left(\cos 2 \pi f_{1} t+\cos 2 \pi f_{2} t\right)
$$

where $f_{1}$ and $f_{2}$ are the frequencies of two wave fields.

In the present case, we employed $f_{1}=87 \mathrm{~Hz}$ and $f_{2}=78 \mathrm{~Hz}$. In this study we extracted the difference frequency response at $\left(f_{1}-f_{2}\right)=$
$9 \mathrm{~Hz}$ because this component is located far apart from the responses at $f_{1}, f_{2}$ and their harmonics which the signal generator might include. Substituting eq 2 into eq 1, we obtain the response at $f_{1}-f_{2}$ as

$$
\begin{gathered}
D\left(f_{1}-f_{2}\right)=\left[\varepsilon_{2} E_{0}{ }^{2}+3 \varepsilon_{4} E_{0}{ }^{4}+\cdots\right] \\
\times \cos 2 \pi\left(f_{1}-f_{2}\right) t
\end{gathered}
$$

The amplitude of $D\left(f_{1}-f_{2}\right)$ includes all the even order dielectric constants. We assumed that the higher order terms $\varepsilon_{4}$ etc. are negligibly small. This assumption was confirmed from the experimental fact that the amplitude of $D\left(f_{1}-f_{2}\right)$ is proportional to the square of the input amplitude $E_{0}{ }^{2}$.

The block diagram of measuring system is shown in Figure 1, which is essentially similar to that by Hayakawa et $a .^{8}$ The system enables us to stimulate the sample by the sum of

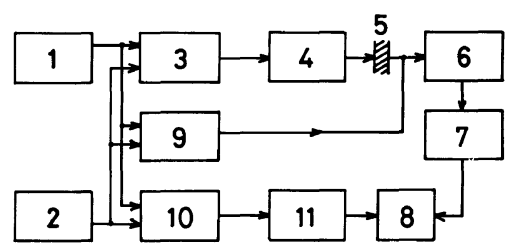

Figure 1. Block diagram of apparatus for second order dielectric constant measurements. 1, oscillator $\left(f_{1}\right) ; 2$, oscillator $\left(f_{2}\right) ; 3$, summing amplifier; 4 , high voltage amplifier; 5 , sample; 6 , charge amplifier; 7 , band pass filter; 8 , osciloscope; 9 , compensator; 10 , multiplier; 11 , band pass filter.

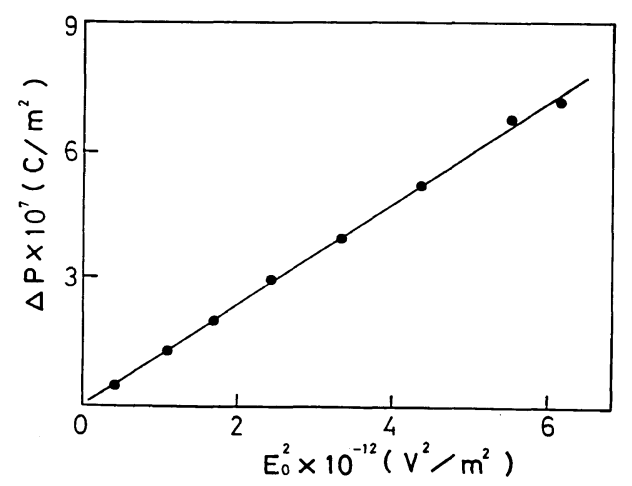

Figure 2. Relation between difference frequency response at $9 \mathrm{~Hz}$ and squared amplitude of input sine waves in poled VDF-TrFE $(52: 48)$ copolymer. 
two waves and extract the difference frequency response from the responses.

The poled sample of the copolymer gives the $\left(f_{1}-f_{2}\right)$ response as illustrated in Figure 2, where we find that the response is proportional to $E_{\mathrm{o}}{ }^{2}$ at least in the range of $E_{0}<2.5 \mathrm{MVm}^{-1}$. Accordingly, we can obtain $\varepsilon_{2}$ from this response.

\section{RESULTS AND DISCUSSION}

Temperature Dependence of Second Order

Dielectric Constant of VDF-TrFE $(52: 48)$

\section{Copolymer}

In this section, the temperature dependence of $\varepsilon_{2}$ of VDF-TrFE $(52: 48)$ copolymer is discussed.

A simple description of elastic Gibbs energy for a ferroelectric material per unit volume is usually written as a function of $P$ as ${ }^{9}$

$$
\begin{aligned}
G-G_{0}= & (1 / 2) \beta\left(T-T_{0}\right) P^{2} \\
& +(1 / 4) \gamma P^{4}+(1 / 6) \delta P^{6}
\end{aligned}
$$

where $G_{0}$ stands for the free energy at $P=0, \beta$, $\gamma, \delta$, and $T_{0}$ are constants. For the first order phase transition, $\beta>0, \gamma<0$, and $\delta>0$, whereas for the second order phase transition, $\beta>0, \gamma>0$, and $\delta>0$.

The $n$-th order non-linear dielectric constant $\varepsilon_{n}$ is defined as

$$
\begin{aligned}
& \varepsilon_{1}=\mathrm{d} P /\left.\mathrm{d} E\right|_{E=0}+\varepsilon_{0}, \\
& \varepsilon_{n}=(1 / n !) \mathrm{d}^{n} P /\left.\mathrm{d} E^{n}\right|_{E=0} \quad(n>2)
\end{aligned}
$$

where $\varepsilon_{0}$ is the permittivity of free space. The second order dielectric constant $\varepsilon_{2}$.is derived from eq 4 as

$$
\varepsilon_{2}=-\left(3 \gamma+10 \delta P_{\mathrm{s}}^{2}\right) P_{\mathrm{s}}\left(\varepsilon_{1}-\varepsilon_{0}\right)^{3}
$$

where $P_{\mathrm{s}}$ is the spontaneous polarization. $\varepsilon_{1}$ and $P_{\mathrm{s}}$ are respectively given by the equations:

$$
\varepsilon_{1}-\varepsilon_{0}=\left[\beta\left(T-T_{0}\right)+3 \gamma P_{\mathrm{s}}^{2}+5 \delta P_{\mathrm{s}}^{4}\right]^{-1}
$$

and

$$
P_{\mathrm{s}}^{2}=(1 / 2 \delta)\left[-\gamma+\left[\gamma^{2}-4 \beta \delta\left(T-T_{0}\right)\right]^{1 / 2}\right]
$$

When the coefficients $\gamma$ and $\delta$ in eq 4 are independent of temperature, the second order dielectric constant $\varepsilon_{2}$ should depend on temperature through $P_{\mathrm{s}}$ and $\varepsilon_{1}$.

The solid line in Figure 3 shows the temperature dependence of $P_{\mathrm{s}}$ in poled VDFTrFE copolymer. $P_{\mathrm{s}}$ gradually decreases with

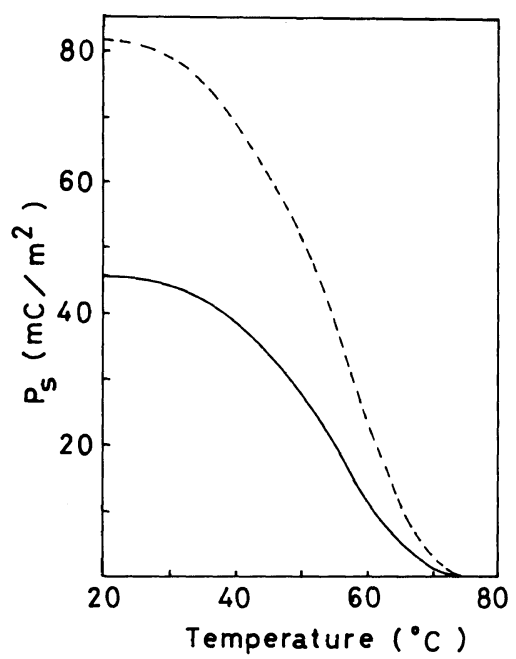

Figure 3. Temperature dependence of spontaneous polarization $P_{\mathrm{s}}$ of poled VDF-TrFE $(52: 48)$ copolymer. Solid line shows $P_{\mathrm{s}}$ of the whole polymer and broken line that of crystal estimated by eq 11 .

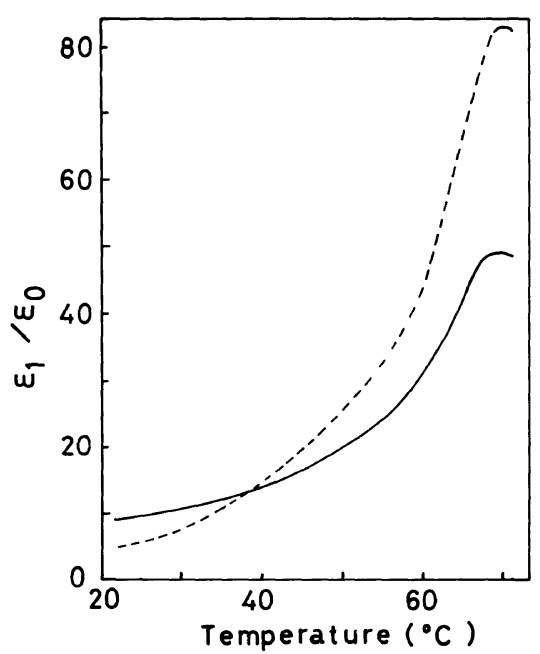

Figure 4. Temperature dependence of linear dielectric constant $\varepsilon_{1}$ of VDF-TrFE $(52: 48)$ copolymer. Solid line shows $\varepsilon_{1}$ of the whole polymer and broken line that of crystal estimated by eq 19 . 


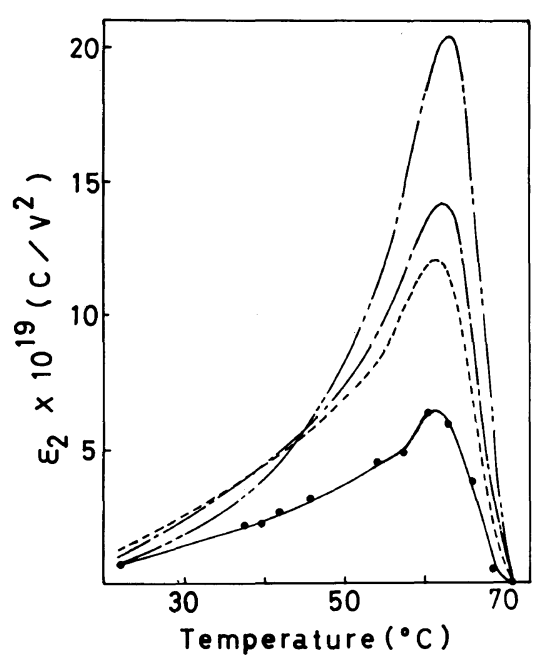

Figure 5. Temperature dependence of second order dielectric constant $\varepsilon_{2}$ of VDF-TrFE $(52: 48)$ copolymer. Solid line shows $\varepsilon_{2}$ of the whole polymer. Broken lines a, b, and c show $\varepsilon_{2}$ of a crystal at $L=0.01,0.1$, and $1 / 3$, respectively.

increasing temperature and vanishes at the transition temperature $T_{\mathrm{c}}$. The temperature dependence of the linear dielectric constant $\varepsilon_{1}$ is illustrated by the solid line in Figure 4. $\varepsilon_{1}$ increases with increasing temperature up to $T_{\mathrm{c}}$. Figure 5 shows the temperature dependence of $\varepsilon_{2} . \varepsilon_{2}$ has a maximum at $61^{\circ} \mathrm{C}$ and vanishes at ca. $70^{\circ} \mathrm{C}$ which corresponds to $T_{\mathrm{c}}$. Vanishment of $\varepsilon_{2}$ at $T_{\mathrm{c}}$ together with $P_{\mathrm{s}}$ can be predicted by eq 6 . The increase of $\varepsilon_{2}$ below $61^{\circ} \mathrm{C}$ is ascribed to the increase of $\varepsilon_{1}$ whereas the decrease of $\varepsilon_{2}$ above $61{ }^{\circ} \mathrm{C}$ to the decrease of $P_{\mathrm{s}}$.

\section{Relation between $\varepsilon_{2}$ and $P_{\mathrm{r}}$}

Consider a system consisting of domains whose spontaneous polarizations are either parallel $\left(+P_{\mathrm{s}}\right)$ or antiparallel $\left(-P_{\mathrm{s}}\right)$ to the electric field. Since the total dipole moment of the system is the sum of dipole moments in each domain, the remanent polarization of the system is written as

$$
P_{\mathrm{r}}=v_{+} \mathrm{P}^{+}+v_{-} \mathrm{P}^{-} \quad\left(\mathrm{P}^{+}=-\mathrm{P}^{-}=P_{\mathrm{s}}\right)
$$

where $v_{+}$and $v_{-}$stand respectively for the volume fractions of parallel and antiparallel

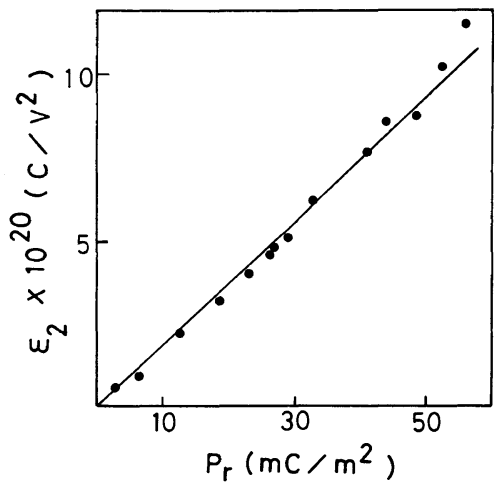

Figure 6. Relation between second order dielectric constant $\varepsilon_{2}$ and residual polarization $P_{\mathrm{r}}$ VDF-TrFE $(52: 48)$ copolymers.

domains. In the range of $E_{0}$ much lower than the coercive field, changes of $v_{+}$and $v_{-}$due to the applied electric field may be neglected. Then $\varepsilon_{2}$ for the composite system is obtained from eq 5 and 9 as

$$
\begin{aligned}
\varepsilon_{2} & =(1 / 2)\left(v_{+}-v_{-}\right) \mathrm{d}^{2} P^{+} /\left.\mathrm{d} E^{2}\right|_{E=0} \\
& =-\left(3 \gamma+10 \delta P_{\mathrm{s}}{ }^{2}\right) P_{\mathrm{r}}\left(\varepsilon_{1}-\varepsilon_{0}\right)^{3}
\end{aligned}
$$

which indicates that $\varepsilon_{2}$ is proportional to the remanent polarization.

Samples with different values of $P_{\mathrm{r}}$ were prepared by decreasing the electric field to zero from intermediate points of the hysteresis curve. Figure 6 shows the $P_{\mathrm{r}}$ dependence of $\varepsilon_{2}$. We can see in Figure 6 that $\varepsilon_{2}$ is proportional to $P_{\mathrm{r}}$ as predicted by eq 10 .

\section{Intrinsic Ferroelectric properties of Polymer Crystal}

The ferroelectric polymer is composed of ferroelectric crystals and paraelectric amorphous region. In order to elucidate the ferroelectric nature of the polymer crystal, the values of $\varepsilon_{1}, \varepsilon_{2}$, and $P_{\mathrm{s}}$ observed for the whole polymer must be converted to those of the crystal by an appropriate model. In the following, $\bar{x}$ stands for a quantity of crystal and $x$ that of the whole polymer.

We adopted the model proposed previously in which the crystal is a prolate ellipsoid with 
the major axis directed along $E$ and is embedded in the polymer matrix of a smearedout mixture of crystals and amorphous region. ${ }^{10,11}$

In this model, the spontaneous polarization and the electric field in the crystal are related to those in the whole polymer by

$$
\bar{P}_{\mathrm{s}}=P_{\mathrm{s}} / B \phi
$$

and

$$
\bar{E}=B E
$$

Here, $\phi$ is the volume fraction of crystals and $B$ is

$$
B=\varepsilon_{1} /\left[\varepsilon_{1}+L\left(\bar{\varepsilon}_{1}-\varepsilon_{1}\right)\right]
$$

where $L$ is the depolarization field coefficient of the crystal.

Since the component at $\left(f_{1}-f_{2}\right)$ in the response arises from the term proportional to $E^{2}$ in eq 1 , the polarization changes at $\left(f_{1}-f_{2}\right)$ in the crystal and the whole polymer are respectively written as

$$
\Delta \bar{P}_{f_{1}-f_{2}}=\bar{\varepsilon}_{2} \bar{E}_{0}^{2} \cos 2 \pi\left(f_{1}-f_{2}\right) t
$$

and

$$
\Delta P_{f_{1}-f_{2}}=\varepsilon_{2} E_{0}^{2} \cos 2 \pi\left(f_{1}-f_{2}\right) t
$$

On the other hand, since the polarization change at $\left(f_{1}-f_{2}\right)$ belongs to the crystal alone, the relation between the polarization changes of the crystal and the whole polymer should be given by the same way as $P_{\mathrm{s}}$ :

$$
\Delta \bar{P}_{f_{1}-f_{2}}=\Delta P_{f_{1}-f_{2}} / B \phi
$$

Equations $12-16$ yield

$$
\bar{\varepsilon}_{2}=\varepsilon_{2} / B^{3} \phi
$$

Similarly, we have in general

$$
\bar{\varepsilon}_{n}=\varepsilon_{n} / B^{n+1} \phi \quad(n>2)
$$

In order to evaluate $B$, the values of $L_{1}, \bar{\varepsilon}_{1}$, and $\varepsilon_{1}$ are required. Since the contribution of amorphous region to $\varepsilon_{1}$ cannot be neglected, $\bar{\varepsilon}_{1}$ is not obtained by eq 18 . The relation between $\bar{\varepsilon}_{1}$ and $\varepsilon_{1}$ is obtained by following pro- cedures. ${ }^{12}$ Consider hypothetical system in which the crystal ellipsoid is replaced by the amorphous phase. Since the sum of this system and our (real) model at volume fractions of $1-\phi$ and $\phi$ should yield a homogeneous whole polymer system, we have

$$
\phi \frac{\bar{\varepsilon}_{1}-\varepsilon_{1}}{\varepsilon_{1}+L\left(\bar{\varepsilon}_{1}-\varepsilon_{1}\right)}+(1-\phi) \frac{\varepsilon_{1 \mathrm{a}}-\varepsilon_{1}}{\varepsilon_{1}+L\left(\varepsilon_{1 \mathrm{a}}-\varepsilon_{1}\right)}=0
$$

where $\varepsilon_{1 \mathrm{a}}$ is $\varepsilon_{1}$ of the amorphous region.

Thus we can estimate $\bar{\varepsilon}_{1}, \bar{\varepsilon}_{2}$, and $\bar{P}_{\mathrm{s}}$ from those of the sample. In this estimation, we assume as the following:

1) $\varepsilon_{1 \mathrm{a}}$ is constant over the temperature range covered in this study.

2) $\bar{\varepsilon}_{1}$ at $20^{\circ} \mathrm{C}$ is equal to $5 \varepsilon_{0}$.

3) The electronic part of $\varepsilon_{1}, \varepsilon_{1 \infty}$, is equal to $4 \varepsilon_{0}{ }^{3}$

4) $\phi=0.55, L=0.01$ and $T_{\mathrm{c}}=68^{\circ} \mathrm{C}$.

First $\varepsilon_{1 \mathrm{a}}$ is evaluated by eq 19 from $\bar{\varepsilon}_{1}$ and $\varepsilon_{1}$ at $20^{\circ} \mathrm{C}$. Then the value of $\bar{\varepsilon}_{1}$ at any temperature is obtained by eq 19 from $\varepsilon_{1 \mathrm{a}}$ and $\varepsilon_{1}$ at the same temperature.

The temperature dependence of $\bar{P}_{\mathrm{s}}, \bar{\varepsilon}_{1}$, and $\bar{\varepsilon}_{2}$ is illustrated by broken lines in Figures $3-5$. In this case, the differences between crystal and whole polymer in the spontaneous polarization and the second order dielectric constant come dominantly from the volume fraction of the crystal because $L$ is small and $B$ is equal almost to unity.

The value of $L$, however, should change with the change in crystal shape, which depends on thermal history or other sample treatments. The present authors found $L$ of poly(vinylidene fluoride) (PVDF) not to be larger than 0.08 from the profile of its $D-E$ hysteresis curve. ${ }^{11}$ Values of $L$ over the range from 0.001 to 0.1 is possible for PVDF and VDF-TrFE copolymers. In Figure $6, \bar{\varepsilon}_{2}$-temperature curves at $L=0.01,0.1$, and 0.33 are illustrated. $\bar{\varepsilon}_{2}$ at $L=0.1$ is approximately $15 \%$ larger than that at $L=0.01$, the latter corresponding to the case of $B$ nearly equal to unity. At $L=0.33$ (spherical crystal model) the 
curve is deformed dominantly by $B$. Since $\bar{\varepsilon}_{n}$ in eq 18 is inversely proportional to $B^{n+1}$, the correction factor by the crystal shape increases with increasing $n$.

Acknowledgments. The authors are greatly indebted to Daikin Kogyo Co. for providing us with samples of VDF-TrFE copolymers. This work was supported in part by a Grantin-Aid on Special Project Research for "Organic Thin Films for Information Conversion" from the Ministry of Education, Science, and Culture of Japan.

\section{REFERENCES}

1. G. T. Davis, M. G. Broadhust, A. J. Lovinger, and T. Furukawa, Ferroelectrics, 57, 73 (1984).

2. M. Jimbo, S. Kobayashi, K. Horino, S. Ikeda, and
Y. Wada, J. Polym. Sci., Polym. Phys. Ed., 22, 2139 (1984).

3. N. Koizumi, N. Haikawa, and H. Habuka, Ferroelectrics, 57, 99 (1984).

4. T. Furukawa, H. Matsuzaki, M. Shiina, and Y. Tazitsu, Jpn. J. Appl. Phys., 24, L661 (1985).

5. S. Ikeda; T. Fukada, M. Jimbo, K. Koyama, and Y. Wada, Jpn. J. Appl. Phys., 24, 865 (1985).

6. K. Tashiro, K. Takano, M. Kobayashi, Y. Chatani, and H. Tadokoro, Ferroelectrics, 57, 297 (1984).

7. A. J. Lovinger, G. T. Davis, T. Furukawa, and M. G. Broadhurst, Macromolecules, 15, 323 (1982).

8. R. Hayakawa, J. Kusahara, K. Hattori, and Y. Wada, Rept. Prog. Polym. Phys. Jpn., 16, 477 (1973).

9. M. E. Lines and A. M. Glass, "Principles and Applications of Ferroelectrics and Related Materials," Clarendon Press, Oxford, 1977, p 71.

10. Y. Wada, Ferroelectrics, 57, 343 (1984).

11. S. Ikeda, M. Jimbo, S. Kobayashi, and Y. Wada, $J$. Polym. Sci., Polym. Phys. Ed., 23, 1781 (1985).

12. R. Landauer, J. Appl. Phys., 23, 779 (1952). 